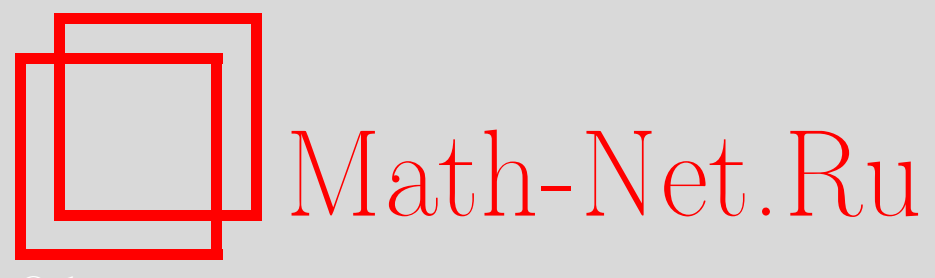

А. Боаг, Л. Радзиловский, Раскраска таблицы в три цвета, Квант, 2021, номер 3, 37-41

DOI: https://doi.org/10.4213/kvant20210304

Использование Общероссийского математического портала Math-Net.Ru подразумевает, что вы прочитали и согласны с пользовательским соглашением http://www . mathnet.ru/rus/agreement

Параметры загрузки:

IP: 3.89 .185 .249

26 апреля 2023 г., 12:11:50

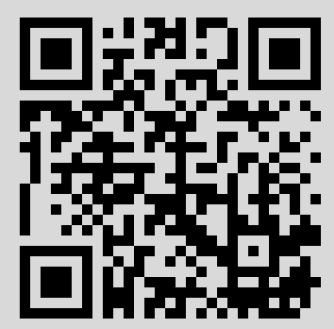




\section{Раскраска таблицы в три цвета}

\section{А.БОАГ, Л.РАДЗИВИЛОВСКИЙ}

$\mathrm{B}$ ЭТОЙ СТАТЬЕ МЫ РАЗБЕРЕМ одну комбинаторную задачу так называемого рамсеевского типа и на ее примере продемонстрируем множество интересных идей и методов - от принципа Дирихле до небольшого путешествия в аффинную геометрию над конечным полем.

Рассмотрим таблицу $m \times n$ ( $m$ строк и $n$ столбцов), где $m, n>1$, и раскраски такой таблицы, при которых каждая клетка покрашена в один из данных $k$ цветов. Назовем раскраску хорошей, если найдется прямоугольник, угловые клетки которого покрашены в один цвет (здесь и далее длина и ширина прямоугольника больше 1). Остальные раскраски назовем плохими. Задача: для фиксированного $k>1$ выяснить, при каких $m$ и $n$ любая раскраска - хорошая. Такие пары $(m, n)$ назовем хорошими, а остальные пары - плохими.

Например, любая пара вида $(k, n)$ всегда будет плохой (напомним, что $k$ - число цветов, а не произвольное число). Действительно, у таблицы $k \times n$ есть плохая раскраска: каждая строка покрашена в свой цвет. С другой стороны, интуиция подсказывает, что пары $(m, n)$, в которых и $m$ и $n$ достаточно велики, должны быть хорошими: в большой таблице прямоугольник какого-то цвета уж точно должен найтись! Сравните с другой классической задачей: среди любых шести людей на земном шаре обязательно найдутся трое попарно знакомых или трое попарно незнакомых. Такого рода вопросы относятся к теории Рамсея. Эта теория изучает условия, при которых в достаточно больших математических объектах обязательно появляется фрагмент данного вида. Другие интересные задачи в духе теории Рамсея можно найти в статье [1].

DOI: https://doi.org/10.4213/kvant20210304
Начнем с простых соображений, верных для любого фиксированного $k$.

- Пары $(m, n)$ и $(n, m)$ хорошие (плохие) одновременно.

- Свойство раскраски быть хорошей или плохой не зависит от перестановок строк и перестановок столбцов.

- Введем порядок на парах:

$$
\begin{aligned}
\left(m^{\prime}, n^{\prime}\right) \geq(m, n) \Leftrightarrow & \left(m^{\prime} \geq m \text { и } n^{\prime} \geq n\right) \\
& \text { или }\left(m^{\prime} \geq n \text { и } n^{\prime} \geq m\right) .
\end{aligned}
$$

Если пара $(m, n)$ хорошая, то хорошие также все пары $\left(m^{\prime}, n^{\prime}\right) \geq(m, n)$.

Уже для четырех цветов нам ответ неизвестен. Для трех цветов мы опишем все хорошие пары. Начнем с более простого случая.

\section{Случай двух цветов}

Пусть цветов всего два: белый и синий. Будем увеличивать число $m$ строк и каждый раз находить наименьшее $n \geq m$, при которых пара $(m, n)$ - хорошая (если такое $n$ существует). Как отмечалось выше, все пары $(2, n)$ плохие.

При $m \geq 3$ в каждом столбце есть хотя бы две клетки одного цвета. Поэтому всякая раскраска с двумя одинаковыми столбцами хорошая. Поскольку всего различных столбцов $2^{m}$, то все пары $(m, n)$, где $m \geq 3, n>2^{m}$, хорошие. В частности, при $m=3$ есть всего 8 разных столбцов высоты 3 , так что пара $(3,9)$ хорошая. Далее, в таблице $3 \times 7$ с различными столбцами обязательно есть одноцветный столбец, скажем синий. Поэтому, если раскраска плохая, то в остальных столбцах может быть максимум одна синяя клетка, а таких столбцов всего 4. Значит, любая раскраска доски $3 \times 7-$ хорошая, т.е. $(3,7)-$ хорошая пара. А пара $(3,6)$ уже плохая, так как даже пара $(4,6)$ плохая: пример плохой раскраски показан на рисунке 1. Столбцы в ней представляют все 6 сочета-

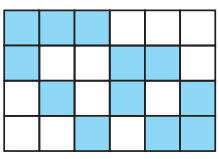

Pис. 1 ний из 4 по 2 (в каждом столбце ровно две синие клетки).

Для полного описания хороших пар осталось исследовать пару $(5,5)$.

Задача 1. Докажите, ито пара $(5,5)$ хорошая (для двух иветов). 
Теорема 1. При раскраске в два цвета хорошими являются в точности следуюuие nары:

$$
(m, n) \geq(3,7),(5,5) .
$$

Далее будем решать задачу для $k=3$. Выведем теорему, аналогичную теореме 1. «Пороговых» пар, аналогичных парам $(3,7)$ и $(5,5)$, будет больше. Теперь случай $m=3$ тривиален: все пары $(3, n)$ плохие. Аналогично случаю двух цветов, пары $(m, n)$, где $m \geq 4$ и $n>3^{m}$, хорошие. Но эта оценка на $n$ слишком грубая. Займемся ее усилением.

\section{Мультиграф раскраски}

Пусть дана некоторая раскраска $K$ таблицы $m \times n$. Сопоставим ей мультиграф $G(K)$ по следующему правилу (в отличие от графа, в мультиграфе две вершины могут соединяться несколькими ребрами). Вершинами в $G(K)$ будут номера строк $1, \ldots, m$ нашей таблицы. Если столбец $X$ пересекает $i$-ю и $j$-ю строки по клеткам одного цвета, то соединим вершины $i$ и $j$ ребром с меткой $X$ (причем если эти две клетки, скажем, синие, то и ребро будем называть синим, однако цвета ребер нас будут редко интересовать). Пример соответствия таблицы и мультиграфа показан на рисунке 2 (белым клеткам

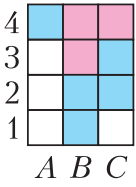

Pис. 2

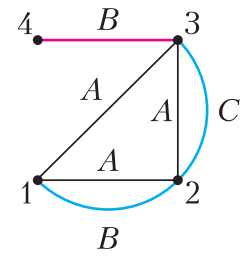

соответствуют черные ребра). Число ребер в полученном мультиграфе обозначим $P(K)$.

Возьмем две строки $i$ и $j$ в какой-нибудь плохой раскраске $K$. Максимум один столбец может пересекать их по белым клеткам, максимум один - по синим и максимум один - по красным. Поэтому в мультиграфе $G(K)$ вершины $i$ и $j$ могут быть соединены максимум тремя ребрами. Поскольку всего пар вершин $C_{m}^{2}=m(m-1) / 2$, мы получаем верхнюю оценку на число ребер:

$P(K) \leq 3 m(m-1) / 2$ для любой

плохой раскраски $K$ таблицы $m \times n$. (2)

А теперь оценим $P(K)$ снизу. Какой бы ни была раскраска $K$ (хорошей или плохой),

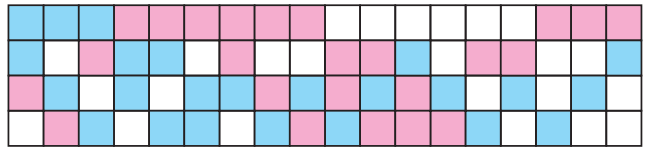

Pис. 3

при $m \geq 4$ в каждом столбце есть две клетки одного цвета. В терминах нашего мультиграфа это значит, что в нем будут ребра, помеченные всеми $n$ столбцами. Значит,

$$
\begin{aligned}
& P(K) \geq n \text { для любой раскраски } K \\
& \text { таблицы } m \times n, \text { где } m \geq 4 . \text { (3) }
\end{aligned}
$$

При $m=4$ оценки (2) и (3) дают неравенство для плохих раскрасок: $n \leq P(K) \leq 18$. Существует ли плохая раскраска при $n=18$ ? Если да, то обе оценки (2) и (3) обращаются в равенства, т.е. в каждом столбце есть клетки всех цветов и любые две строки имеют ровно одну общую клетку каждого цвета. Один из примеров показан на рисунке 3. Итак, пара $(4, n)$ - хорошая тогда и только тогда, когда $n \geq 19$.

\section{Неравенство между числом столбцов и числом ребер}

Усилим неравенство (3), дающее хорошую оценку лишь при $m=4$. Для этого точнее оценим вклад каждого столбца в общее число ребер. Для примера рассмотрим таблицу на рисунке 4.

Столбец $A$ «соединяет» все $C_{5}^{2}=10$ пар строк, столбец $B-$

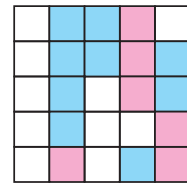

$A B C D E$ Pис. 4 $C_{4}^{2}=6$ пар строк (с 1 по 4), столбец $C$ $3+1=4$ пары, столбец $D-$ три пары, столбец $E$ - две пары. $C$ точностью до переименования цветов и порядка строк мы перебрали все варианты столбцов высоты 5. Как видим, каждый соединяет хотя бы две пары строк, а потому $P(K) \geq 2 n$ при $m=5$.

Вообще, пусть в столбце высоты $m$ имеется $x$ белых, $y$ красных и $z$ синих клеток. Тогда он соединяет

$$
\begin{aligned}
C_{x}^{2}+C_{y}^{2}+C_{z}^{2}= \\
\quad=(x(x-1)+y(y-1)+z(z-1)) / 2
\end{aligned}
$$

строк. Нас интересует минимум $f(m)$ этого выражения при условии $x+y+z=m$ : раз в каждом из $n$ столбцов хотя бы $f(m)$ пар одинаково раскрашенных клеток, то в муль- 
тиграфе ребро, помеченное любым из $n$ столбцов, встретится хотя бы $f(m)$ раз. Это дает оценку

$$
P(K) \geq n f(m) \text { для любой раскраски } K
$$$$
\text { таблицы } m \times n \text {, где } m \geq 4 \text {. (5) }
$$

С учетом обратной оценки (2) отсюда заключаем, что

все пары $(m, n)$, где $m \geq 4$,

$$
n>[3 m(m-1) / 2 f(m)],- \text { хорошие }
$$

(здесь квадратными скобками обозначена целая часть числа).

Интуиция и пример с $m=5$ подсказывают, что минимум $f(m)$ выражения (4) достигается, когда числа $x, y, z$ «примерно равны». Идея в том, что если какие-то два из этих чисел сильно различаются, то их можно сблизить с сохранением суммы, отчего значение выражения (4) уменьшится (это иллюстрирует рисунок 5). Кстати, это общее свойство выпуклых функций. Сформулируем

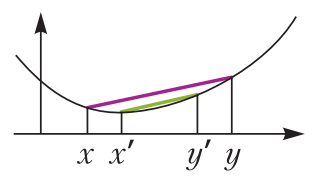

Pис. 5 четкое утверждение и предложим читателю его аккуратно доказать. Благо мы имеем дело с совсем простой, квадратичной функцией.

Задача 2. Докажите, что минимум выражения (4) при условии $x+y+z=m$ достигается, когда числа $x, y, z$ отличаются не более чем на 1 ( $x, y, z$ - челые неотрицательные числа).

Такое разбиение числа $m$ назовем равномерным.

Составим таблицу:

\begin{tabular}{|c|c|c|c|}
\hline$m$ & $\begin{array}{c}\text { Равномерное } \\
\text { разбиение } \\
m=x+y+z\end{array}$ & $f(m)$ & {$\left[\frac{3 m(m-1)}{2 f(m)}\right]$} \\
\hline 4 & $2+1+1$ & 1 & 18 \\
\hline 5 & $2+2+1$ & 2 & 15 \\
\hline 6 & $2+2+2$ & 3 & 15 \\
\hline 7 & $3+2+2$ & 5 & 12 \\
\hline 8 & $3+3+2$ & 7 & 12 \\
\hline 9 & $3+3+3$ & 9 & 12 \\
\hline 10 & $4+3+3$ & 12 & 11 \\
\hline 11 & $4+4+3$ & 15 & 11 \\
\hline
\end{tabular}

Мы остановились на $m=11$, так как верхняя граница для $n$ тогда тоже 11. Мы бы завершили решение, если бы привели при- меры плохих раскрасок размеров $6 \times 15,9 \times 12$ и $11 \times 11$. Первые два примера мы построим и покажем, как до них догадаться, а вот с последним случаем все не так просто. Но обо всем по порядку.

\section{Пример $6 \times 15:$ турнирная таблица}

Поскольку минимум $f(6)=2$ достигается лишь на равномерном разбиении $6=2+2+2$, то в плохой раскраске $6 \times 15$ каждый столбец содержит по две клетки каждого цвета, причем одна и та же пара клеток в разных столбцах не может быть покрашена одним цветом. Такую таблицу можно интерпретировать как турнирную для своеобразного троеборья между шестью участниками. Строки таблицы - игроки $1, \ldots, 6$. Каждый столбец - это раунд, в котором белые клетки обозначают играющих в шахматы, красные - в шашки, синие - в нарды. При этом за весь турнир каждая пара играет между собой в каждую игру по разу. Как раз получается $15=5 \times 3$ раундов: каждый играет с пятью другими в три игры. Как же составить такую таблицу?

Начнем с построения более простой таблицы - для шахматного турнира между шестью игроками, имеющими три доски. Нужно разбить их на пары пятью способами, чтобы каждый побывал в паре с каждым ровно один раз. Для этого есть простая геометрическая картинка. Возьмем вершины правильного пятиугольника и его центр всего 6 точек, они обозначают участников. Эти точки разбиваются ровно пятью способами на такие пары: сторона, параллель-

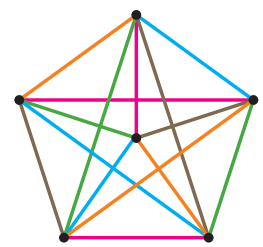

Pис. 6 ная ей диагональ, две оставшиеся точки (рис.6).

Задача 3. Обобщите описанную конструкцию на любое число шахматистов $2 n$ или $2 n+1$, имеющих $n$ досок и желающих провести однокруговой турнир за наименьшее число туров.

Теперь превратим таблицу $6 \times 5$ для шахматного турнира в искомую таблицу $6 \times 15$ для троеборья. Каждый столбец шахматной таблицы - это просто разбиение на пары $A$, $B$ и $C$. Объявим, что в первом туре пара $A$ играет в шахматы, пара $B$ - в шашки, пара $C$ - в нарды. Во втором туре те же пары 


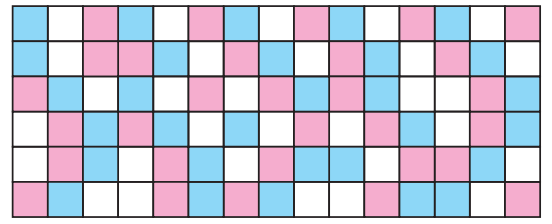

Pис. 7

играют в игры, сдвинутые по циклу: $A-$ в шашки, $B-$ в нарды, $C-$ в шахматы. Наконец, в третьем туре сделаем цикл еще раз: $A-$ в нарды, $B-$ в шахматы, $C-$ в шашки. Проделав эту процедуру с каждым столбцом шахматной таблицы $6 \times 5$, получим искомую раскраску $6 \times 15$, пример приведен на рисунке 7.

\section{Пример $9 \times 12$ : конечная аффинная геометрия}

В плохой таблице $9 \times 12$ в каждом столбце должно быть по три клетки каждого цвета (минимум $f(9)=12$ достигается лишь на разбиении $9=3+3+3)$. Построить такую таблицу поможет аффинная геометрия над полем $\mathbb{Z}_{3}=\{-1,0,1\}$. Пугаться не стоит. Сейчас все объясним по-простому.

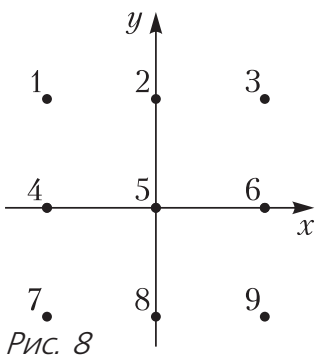

Сопоставим каждой строке точку $(x, y)$ на плоскости с координатами $x, y \in\{0,1,-1\}$, которые складываются, вычитаются и умножаются по модулю 3 (рис.8). Например, $1+1=-1$, $(-1)(-1)=1$. Прямые, как обычно, задаются линейными уравнениями вида $y=a x+b$ и $x=c\left(a, b, c \in \mathbb{Z}_{3}\right)$. Каждая прямая, тем самым, состоит из трех точек. Параллельные прямые - те, которые не пересекаются, т.е. вертикальные $x=b$, а также прямые первого вида с одинаковым угловым коэффициентом $a$ и разными коэффициентами $b$. Всего получается 12 прямых, разбитых на 4 группы параллельных (4 направления). Возьмем любое такое разбиение на параллельные прямые и покрасим их в белый, синий и красный цвета произвольным образом. Перенеся цвет каждой точки в соответствующую ей клетку таблицы $9 \times 4$, получим плохую раскраску. И даже «очень плохую» - в том смысле, что эта раскраска обладает более сильным свойством: если в каком-то прямоугольнике угловые клетки в одном столбце одноцветны, то угловые клетки другого столбца обязательна разноцветны.

Действительно, парам клеток каждого из четырех столбцов отвечают пары точек на соответствующем рисунке с параллельными прямыми. Если на одном рисунке точки одноцветны, то они лежат на одной из параллельных прямых, но тогда на другом рисунке те же точки лежат на разных параллельных прямых, а значит, покрашены в разные цвета.

Теперь сделаем из нашей таблицы $9 \times 4$ плохую таблицу $9 \times 12$ : для каждого столбца сделаем две копии и сдвинем в них цвета по циклу синий $>$ белый $>$ красный $>$ синий

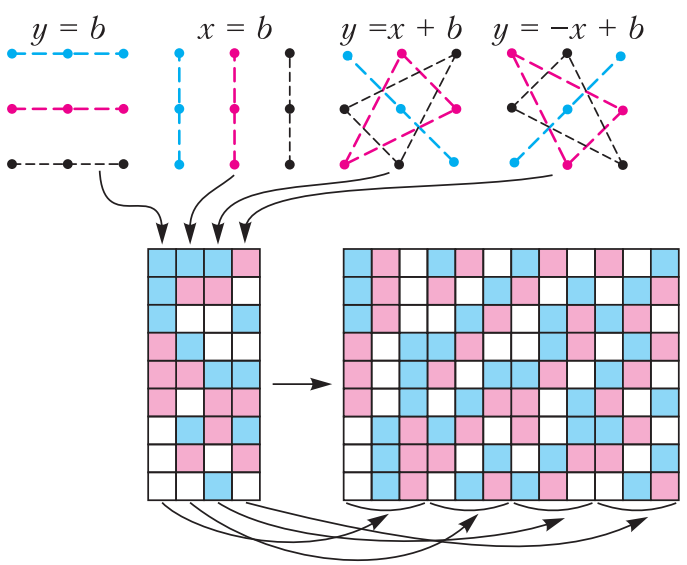

Рис. 9

(рис.9). Раскраска и впрямь плохая: если прямоугольник взят из одной группы скопированных столбцов, то его левые и правые углы покрашены в разные цвета, а иначе для него выполняется свойство (7).

Кстати, метод, которым мы построили таблицу $9 \times 4$, используют для организации турнира со следующими условиями. В турнире всего $q^{2}$ участников, где $q$ - степень простого числа (у нас $q=3$ ). В каждом туре участники как-то разбиваются на $q$ групп по $q$ в каждой, внутри группы каждый играет с каждым, при этом за весь турнир каждый играет с каждым ровно один раз. Чтобы составить расписание разбиений на группы, используют описанную выше конструкцию с полем из $q$ элементов вместо $\mathbb{Z}_{3}$. При простом $q$ это просто поле вычетов по модулю $q$. Подробнее об этом рассказано в статье [2]. 
Анализ таблиц $10 \times 10$ и $10 \times 11$

Оказывается, примера плохой раскраски $11 \times 11$ не существует, т.е. пара $(11,11)$ хорошая. На самом деле, даже пара $(10,11)$ хорошая, но для доказательства недостаточно оценки (5). Приведем план доказательства, оставив детали читателю в виде задачи. Предположим, нам удалось построить плохую раскраску $K$ размера $10 \times 11$. Ребер каждого цвета в мультиграфе $G(K)$ не более $C_{10}^{2}=45$. Клеток какого-то цвета, скажем синего, в $C$ хотя бы 37 ( так как $36 \times 3=108$ ). Если их ровно 37 и они распределены по столбцам равномерно, то среди них 4 четверки и 7 троек, и тогда синих ребер ровно $4 C_{4}^{2}+7 C_{3}^{2}=45$.

Задача 4. Докажите, что при любом другом распределении синих клеток по столбиам синих ребер было бы больше.

Итак, можно считать, что в столбцах $A-D$ по 4 синих клетки, а в остальных - по 3 .

Задача 5. Докажите, что среди столбцов $A-D$ :

a) любые два имеют ровно одну общую (с тем же номером) синюю клетку;

б) никакие три не имеют общей синей клетки.

Таким образом, можно считать, что синие клетки в столбцах $A-D$ расположены, как на рисунке 10. Галочками отмечены строки, в которых две синие клетки. Эти строки назовем темными, а остальные - свет-

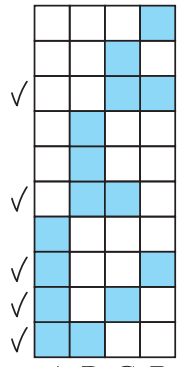

$A B C D$ Рис. 10 лыми. Попробуем покрасить в одном из оставшихся столбцов три клетки синим цветом, чтобы не получилось синего прямоугольника.

Задача 6. Докажите, что при этом хотя бы две из трех синих клеток будут в светльх строках.

Поскольку светлых строк всего 4, то из них можно образовать лишь $C_{4}^{2}=6$ пар, поэтому среди семи последних столбцов обязательно найдутся два с общей парой синих клеток. Итак, любая таблица $10 \times 11$ хорошая.

Пример плохой таблицы $10 \times 10$ мы нашли с помощью компьютера (рис. 11).

Собирая все вместе, получаем следующую теорему.

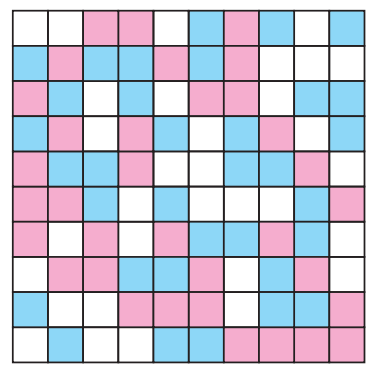

Рис. 11

Теорема 2. При раскраске в три чвета хорошими являются в точности следующие nары:

$$
(m, n) \geq(4,19),(6,16),(9,13),(10,11) .
$$

Задача 7. Рассмотрим такую же задачу для $k$ иветов. Докажите, что пары $\left(k+1, k^{2}(k+1) / 2\right) u\left(k^{2}+k, k^{2}+k\right)$ хорошие.

Задача 8. Пусть $q$ - степень простого числа. Докажите, что для q иветов пара $\left(q^{2}, q^{2}+q\right)$ плохая. (Если вы не знаете других конечных полей, кроме полей вычетов, считайте, что $q$ - простое.)

\section{Литература}

1. А.Райгородский. Задачи о пересечениях множеств. - «Квант», 2016, №5-6.

2. М.Горелов. Абстрактная математика волейбольного турнира. - «Квант», 2015, №2.

\section{(Начало см. на с. 34)}

Ясно, что наступит момент, когда этот центр масс окажется слева от точки $О$. С поступлением этой последней капельки бак с водой начнет опрокидываться. И чем дальше, тем быстрее. Вот это явление потери устойчивости и называется катастрофой.

\section{Задачи для самостоятельного решения}

1. Один и тот же квадратный бак, стоящий на наклонной плоскости, в одном случае упирается в бортик ребром основания, а в другом углом основания (ребра основания образуют угол $45^{\circ}$ с бортиком). В каком случае бак более устойчив?

2. В школьном саду квадратный бак, стоящий на наклонной плоскости, заполняли водой до тех пор, пока вода не стала выливаться из бака. Толик Втулкин утверждает, что в этот бак, не убирая его с этой плоскости, можно влить еще больше воды. Может ли утверждение Толика Втулкина быть верным? 